\title{
The Spatial Distribution of Perseverations in Neglect Patients during a Nonverbal Fluency Task Depends on the Integrity of the Right Putamen
}

\author{
Kaufmann B C**a ${ }^{a}$, Frey $\mathrm{J}^{*}{ }^{a}$, Pflugshaupt $\mathrm{T}^{a}$, Wyss $\mathrm{P}^{\mathrm{c}}$, Paladini $\mathrm{R} \mathrm{E}^{b, c}$, Vanbellingen $\mathrm{T}^{a, c}$, Bohl- \\ halter $\mathrm{S}^{a}$, Chechlacz $\mathrm{M}^{d}$, Nef $\mathrm{T}^{c}$, Müri RM ${ }^{b, c}$, Cazzoli $\mathrm{D}^{b, c}$, Nyffeler $\mathrm{T}^{a, b, c}$. \\ * contributed equally to the paper
}

\footnotetext{
${ }^{a}$ Neurocenter, Luzerner Kantonsspital, Lucerne, Switzerland

${ }^{b}$ Perception and Eye Movement Research Laboratory, Department of Clinical Research and Department of Neurology, Inselspital, Bern, Switzerland

${ }^{c}$ ARTORG Center for Biomedical Engineering Research, University of Bern, Bern, Switzerland

${ }^{\mathrm{d}}$ University of Birmingham, School of Psychology, Birmingham, United Kingdom
}

Corresponding author:

Prof. Dr. med. Thomas Nyffeler

ARTORG Center for Biomedical Engineering Research,

University of Bern, Bern, Switzerland

Neurocenter,

Luzerner Kantonsspital, Lucerne, Switzerland

$\underline{\text { Tel. }+41412055686}$

thomas.nyffeler@luks.ch 


\section{Abstract}

Deficient inhibitory control leading to perseverative behaviour is often observed in neglect patients. Previous studies investigating the relationship between response inhibition and visual attention have reported contradictory results: some studies found a linear relationship between neglect severity and perseverative behaviour whereas others could not replicate this result. The aim of the present study was to shed further light on the interplay between visual attention and response inhibition in neglect, and to investigate the neural underpinnings of this interplay. We propose the use of the FivePoint Test, a test commonly used to asses nonverbal fluency, as a novel approach in the context of neglect . In the Five-Point Test, participants are required to generate as many different designs as possible, by connecting dots within forty rectangles. We hypothesised that, because of its clear definition of perseverative errors, the Five-Point Test would accurately assess both visual attention as well as perseverative behaviour. We assessed 46 neglect patients with right-hemispheric stroke, and performed voxel-based lesion-symptom mapping (VLSM) to identify neural substrates of perseverative behaviour as well as the spatial distribution of perseverations. Our results showed that the Five-Point Test can reliably measure neglect and perseverative behaviour. We did not find any significant relationship between neglect severity and the frequency of perseverations. However, within the subgroup of neglect patients who displayed perseverative behaviour, the spatial distribution of perseverations significantly depended on the integrity of the right putamen. We discuss the putative role of the putamen as a potential subcortical hub to modulate the complex integration between visual attention and response inhibition processes.

Keywords: Neglect; Visual Attention; Response Inhibition; Five-Point Test; Lesion mapping; Putamen 


\section{Introduction}

Visual attention and response inhibition are strongly interrelated in everyday behaviour. The former is crucial for the monitoring of environmental signals (Bari \& Robbins, 2013) and the detection of relevant changes. Response inhibition, on the other hand, allows to flexibly adjust behaviour in response to these changes (Bari \& Robbins, 2013; van Belle, Vink, Durston, \& Zandbelt, 2014). At the cortical level, a current model suggests that visual attention is controlled by a ventral attention network - which includes the inferior parietal lobule (IPL), the superior temporal gyrus (STG), and the inferior frontal gyrus (IFG) - and by a dorsal attention network, including the medial intraparietal sulcus (mIPS), the superior parietal lobule (SPL), the precuneus, the supplementary eye field (SEF), and the frontal eye field (FEF) (Corbetta \& Shulman, 2002; Karnath \& Rorden, 2012). The distinct ventral and dorsal attention networks have collaborative roles, allowing flexible adjustment of their dynamic interaction (Vossel, Geng \& Fink, 2014). Response inhibition, on the other hand, is controlled by a cortical network including the inferior frontal gyrus (IFG), the dorsolateral prefrontal cortex (DLPFC), the cingulate cortex, and the premotor cortex (Gandola, et al., 2013; Husain \& Kennard, 1997; Mannan, et al., 2005; Menon, Adleman, White, Glover, \& Reiss, 2001; Pierrot-Deseilligny, Rivaud, Gaymard, \& Agid, 1991). Despite the extensive literature concerning visual attention and response inhibition, theories about these two cognitive functions have mostly been developed separately. Even less is known about how both functions interact, i.e., how visual attention influences response inhibition. One approach to analyse this topic is to assess the behaviour of patients suffering from an impairment of these cognitive functions due to stroke. A lesion involving the attentional network may lead to neglect (i.e., the failure to attend to the contralesional hemispace), whereas another lesion involving the response inhibition network may lead to perseverative behaviour, defined as a failure to inhibit prepotent responses and/or their extension to different behaviours (Jahanshahi, Obeso, Rothwell, \& Obeso, 2015; Pia, Folegatti, Guagliardo, Genero, \& Gindri, 2009). However, strokes do not follow functional anatomy, but vascularisation, so that especially after extensive strokes (e.g. large MCA strokes) both networks may be damaged at the same 
time. Indeed, in stroke patients with neglect, perseverations are frequently observed, occurring in 30\% (Na, et al., 1999) to 90\% (Rusconi, Maravita, Bottini, \& Vallar, 2002; Vallar, Zilli, Gandola, \& Bottini, 2006) of cases.

According to Rusconi et al. (2002) and Vallar et al. (2006), neglect and perseverative behaviour can co-occur, but represent two independent disorders, both functionally and anatomically. For instance, in cancellation tasks - where perseverative behaviour in neglect patients can take the form of erroneous re-cancellations of ipsilesional targets or distractors; Mark, Kooistra, \& Heilman, 1988; Vallar, et al., 2006 - double dissociations between contralesional omissions and perseverative errors have been documented (i.e., some patients show contralesional omissions, but no perseverations, and other patients show the reverse behavioural pattern; Na, et al., 1999; Nys, van Zandvoort, van der Worp, Kappelle, \& de Haan, 2006; Pia, et al., 2009; Ronchi, Posteraro, Fortis, Bricolo, \& Vallar, 2009; Rusconi, et al., 2002). Moreover, some studies have shown that the number of perseverative errors does not seem to correlate with neglect severity (Pia, et al., 2009; Pia, Ricci, Gindri, \& Vallar, 2013; Rusconi, et al., 2002; Vallar, et al., 2006). However, it has also been shown that visual attention impairment critically influences the number of perseverations. For instance, several studies in patients with neglect demonstrated that perseveration severity is related to neglect severity, and that the amount of perseverative responses linearly increases towards the ipsilesional side of space (Mannan, et al., 2005; Na, et al., 1999; Nys, et al., 2006). Others, in turn, have suggested that the highest degree of perseveration is found in patients with mild to moderate neglect severity, the interaction between neglect and perseveration following an "inverted U-curve" (Kleinman, DuBois, Newhart, \& Hillis, 2013).

The discrepancy between the aforementioned results might be explained, at least in part, by the heterogeneous assessment methods and analysis techniques applied the different studies. For instance, in cancellation tasks, the assessment of the absolute number of perseverations might lead to biased results, since neglect patients often do not cancel any targets at all within the left, contralesional side of space (e.g. Rusconi et al., 2002). Furthermore, no univocal definition of perseverative errors 
has been used in previous studies using cancellation tasks (for an overview, see Gandola et al., 2013), leading to very different forms of drawing behaviour being considered as perseverative (i.e., "scribbling" outside of a target, drawing additional targets, drawing cartoons, etc.).

The aim of the present study is to shed further light on the interplay between visual attention and response inhibition in neglect patients, by using a novel assessment that has the potential to measure the spatial deployment of visual attention and perseverative behaviour more accurately. To this end, we used the Five-Point Test (Regard, Strauss, \& Knapp, 1982), a sensitive neuropsychological measure of figural fluency, in which perseverative errors are clearly defined. Participants are given three minutes time to generate as many different designs as possible by connecting at least two out of five dots with straight lines. Repeated designs are regarded as perseverative errors. We hypothesized that this test would represent a sensitive instrument to assess both spatial biases in visual attention and perseverative behaviour in neglect patients. Moreover, we aimed at investigating the neural correlates subtending the interaction between visual attention and response inhibition, using voxel-based symptom-lesion mapping (VLSM). 


\section{Methods}

\subsection{Subjects}

Forty-six patients suffering from left-sided visual neglect after a first, ischemic or haemorrhagic, right-hemispheric stroke (aged between 27 and 82, mean $=60.54, S D=13.58 ; 20$ women; mean years of education $=12.05, S D=3.19$ ) were included in the study after giving written, informed consent. Figure 1 shows an overlap map of the lesions of all patients included in the study. Diagnosis of neglect was based on performance in the following tasks (all printed on A3 sheets of paper, in landscape orientation): (1) The Line Bisection Task (Wilson, Cockburn, \& Halligan, 1987). A mean relative rightward deviation equal to or greater than $11 \%$ from the actual midline was considered as clinically relevant (Wilson, et al., 1987); (2) A cancellation task, i.e., The Bells test (Gauthier, Dehaut, \& Joanette, 1989), the Star Cancellation test (Wilson, et al., 1987), or the Random Shape Cancellation test (Weintraub \& Mesulam, 1988). The Centre of Cancellation (CoC), i.e., the centre of mass of the spatial distribution of detected items, was used to assess neglect (Rorden \& Karnath, 2010). The CoC allows quantifying neglect severity taking into account both the number of omissions and the spatial distribution of these omissions (Rorden \& Karnath, 2010). Furthermore, calculating the $\mathrm{CoC}$ also allows comparing the same indicator across the different cancellation tasks. A $\mathrm{CoC}$ value of 0 indicates an unbiased spatial distribution; positive $\mathrm{CoC}$ values indicate a shift towards the right side of space, while negative $\mathrm{CoC}$ values indicate a shift towards the left side of space. $\mathrm{CoC}$ values larger than 0.08 were considered as clinically relevant (C. Rorden \& H.O. Karnath, 2010). Patients who fulfilled the criterion for clinical significance in at least one of the tests were considered as presenting with visual neglect, and were thus included in the study.

Twenty healthy controls were matched to the patient group with respect to age, sex, and years of education (aged between 51 and 82, mean $=65.75, S D=9.05 ; 11$ women; mean years of education $=12.55, S D=2.24)$. There was no statistically significant difference between the patient and the healthy control groups with respect to age $(t(64)=1.567, p=.122 ; 2$-tailed $)$, gender $(\chi 2(1)=.743, p$ 
$=.389)$, or education $(t(64)=.630, p=.531 ; 2$-tailed $)$. All participants had normal or corrected-tonormal visual acuity.

Ethical approval to conduct the present study was provided by the Ethics Committees of the States of Bern and Lucerne. The study was carried out in accordance with the principles laid down in the latest version of the Declaration of Helsinki.

Figure 1

about here

\subsection{Five-Point Test}

In the Five-Point Test (Regard, et al., 1982), participants were presented with an A4 sheet of paper, in portrait orientation, on which a grid composed of 40 rectangles was printed ( 5 rectangles within each line and 8 rectangles within each column of the grid; size of all rectangles $34 \mathrm{~mm}$ x $28 \mathrm{~mm}$ each). Each rectangle contained a fixed pattern of five dots, symmetrically arranged (see Figure 4 for a depiction of the test grid). Participants were required to generate as many different designs as possible, one in each rectangle, by connecting at least two out of the five dots with straight lines. Repeated designs (i.e., drawing exactly the same pattern of lines multiple times) were regarded as perseverative errors. Designs with lines that failed to connect dots were regarded as rule violations and were not scored. The number of unique designs and of perseverative errors generated during three minutes was scored. 


\subsection{Analyses of Behavioural Data}

\subsubsection{Figural Fluency Performance and Spatial Distribution of Designs in the Five-Point Test}

To assess participants' performance in the Five-Point Test, figural fluency (i.e., the number of all produced designs) and the spatial distribution of the designs (i.e., the $\mathrm{CoC}$ of all designs) were compared between neglect patients and healthy controls, using an independent samples $t-$ test.

An additional analysis aimed at examining more specifically the spatial distribution of the produced designs over the five columns of the Five-Point Test grid in the two groups of participants. For this purpose, we performed a mixed-design analysis of variance (ANOVA) with the between-subjects factor 'group' (healthy controls and neglect patients) and the within-subjects factor 'Column' (columns of the test grid, numbered one to five). Homogeneity of variances was tested using Mauchly's test of sphericity. If the sphericity condition was not met, the degrees of freedom were adapted by means of the Huynh-Feldt correction.

For all analyses, a $p$-value of $<.05$ was considered as statistically significant. For all $t$-tests, the Levene's test was used to assess homogeneity of variances. If the condition concerning the homogeneity of variances was not met, the degrees of freedom were corrected by means of the WelchSatterthwaite method (as implemented in SPSS 23).

\subsubsection{The Five-Point Test as a Diagnostic Tool for the Evaluation of Neglect}

In order to assess whether the Five-Point Test represents a valid diagnostic tool for the evaluation of neglect and its severity, a second series of analyses aimed to compare the results of the Five-Point Test with the ones of commonly used cancellation tasks and the Line Bisection Task. Therefore, the CoCs of the Five-Point Test and the $\mathrm{CoC}$ of the cancellation tasks as well as the $\mathrm{CoC}$ of the FivePoint Test and the patients' performance obtained from the Line Bisection Task were correlated using a Pearson's correlation. In addition, to compare the sensitivity of the two types of tests, the 
CoCs obtained from the cancellation tasks and from the Five-Point Test in neglect patients were compared using a paired sample $t$-test.

In order to assess the relationship between the spatial distribution of designs and the number of produced designs in neglect patients, the CoCs obtained from the Five-Point test were correlated with the number of produced designs using a Pearson's correlation.

\subsubsection{Perseverative Behaviour in the Five-Point Test}

An additional independent samples $t$ - test was used to test whether the percentage of perseverative errors differed significantly between patients and healthy controls.

In order to examine if neglect patients who produced a higher number of correct designs in the Five-Point Test showed a higher number of perseverative errors in the same test, Pearson's correlation was calculated between both variables.

Whether patients with more severe neglect would also show a larger amount of perseverative errors was examined with a Pearson's correlation between the CoC of the Five-Point Test (including all items) and the percentage of perseverative errors.

In order to analyse if the increased perseverative behaviour in neglect patients was biased by neglect itself - i.e. patients might not be able to decide whether two designs are the same or different because of their neglect - the mean number of Neglec-driven perseverations and the mean number of Non-Neglect-driven perseverations was compared within the Neglect group using a paired t-test. Neglect-driven perseverations were defined as perseverations that occur in a column that is on the right-hand of a previous design with the same pattern. Hereby the patient might not be able to perceive whether two designs are the same or different because he might not attend to the pattern he has drawn previously (Figure 2). On the other hand, Non-Neglect-driven perseverations were defined as perseverations that occur in a column that is on the left-hand or below of a previous design with the same pattern (Figure 2). Hereby the possibility of seeing the original pattern should not be limited by the neglect itself. 
Figure 2

about here

\section{Temporal Distribution of Perseverations}

Additionally, we aimed at assessing the temporal distribution of perseverations in detail. In patients with perseverations $(n=32)$ the mean number of continuous perseveration was compared to the mean number of recurrent perseverations using a paired t-test. Based on the taxonomy of Sandson and Albert (1987) we defined continuous perseverations as repetitive designs in directly adjoining rectangles to the same designs. Recurrent perseverations were defined as repetitive designs in not adjoining rectangles (Figure 3).

Figure 3

about here

A descriptive analysis aimed to judge the overall variability of perseverative designs. Therefore, the mean number as well as the distribution of different perseverative designs was analysed within neglect patients.

\section{Spatial Distribution of Perseverations}

Furthermore, we aimed at assessing the spatial distribution of perseverations in closer detail. First, in order to analyse whether the spatial distribution of unique designs and perseverative designs 
would differ in neglect patients with perseverative behaviour, we compared the $\mathrm{CoC}$ of unique designs with the $\mathrm{CoC}$ of perseverations by means of a dependent sample $t$-test.

In a second step, and since some patients showed a stronger rightward shift in the production of perseverations than in the production of unique designs (i.e., a higher $\mathrm{CoC}$ of perseverations compared to the $\mathrm{CoC}$ of unique designs), we divided patients presenting perseverations into two subgroups: 1) patients with no rightward shift in perseverations production (i.e., CoC perseverations $\leq$ CoC unique designs; see Figure 4A for an example); and, 2) patients with a rightward shift in perseverations production (i.e., $\mathrm{CoC}$ perseverations $>\mathrm{CoC}$ unique designs; see Figure $4 \mathrm{~B}$ for an example).

Figure 4

about here

\subsection{Lesion Mapping and Analysis}

\subsubsection{Lesion mapping}

Lesions were manually delineated on the patients' individual structural MRI images by an experienced rater, by means of the MRIcron software (http://sph.sc.edu/comd/rorden/mricron). The rater was naïve with respect to the hypotheses of the study. The same lesion delineation procedure outlined by Karnath and colleagues (Karnath, Rennig, Johannsen, \& Rorden, 2011) was applied, i.e., diffusion-weighted scans were used for lesion mapping if an MRI was conducted within the first 48 h post-stroke, otherwise T2-weighted scans were used. The borders of the lesions were manually delineated on every transverse slice of the individual MRI images. Images were then normalised 
into MNI space with the Clinical Toolbox for SPM (Rorden et al., 2012;

https://www.nitrc.org/projects/clinicaltbx/), using enantiomorphic normalization (Nachev et al., 2008) and run in SPM12 (http://www.fil.ion.ucl.ac.uk/spm).

\subsubsection{Lesion overlap and subtraction, and lesion-symptom mapping analyses}

The freely available NPM software (http://www.cabiatl.com/mricro/npm/) was used for voxel-based lesion-symptom mapping (VLSM). The Bruner-Munzel test was chosen for analyses of continuous behavioural data (Rorden, Karnath, \& Bonilha, 2007), using the CoC of all designs as predictor $(n=$ 46). For lesion comparisons between neglect patients with perseverations $(n=32)$ and without perseverations $(n=14)$, the Liebermeister test was applied. Furthermore, subtraction plots were computed (i.e., subtracting the lesions of neglect patients with perseverations minus the lesions of neglect patients without perseverations), by means of the MRIcron software.

An additional VLSM analysis was computed in order to asses lesions in the context of temporal distribution of perseverations. Therefore VLSM analysis was computed in order to compare the lesions of neglect patients with continuous $(n=5)$, and those with recurrent perseverations $(n=10)$, using the Liebermeister test.

Furthermore, VLSM analysis was computed in order to compare the lesions of neglect patients with and those without a rightward shift in perseveration production, again using the Liebermeister test.

For all analyses, only voxels that were lesioned in at least $20 \%$ of the patients were included. The significance threshold was adjusted by means of a false discovery rate (FDR criterion of 0.05). Furthermore, as proposed by Medina and colleagues (Medina, Kimberg, Chatterjee, \& Branch Coslett, 2010), we controlled for multiple comparisons using a permutation-based thresholding (Kimberg, Coslett, \& Schwartz, 2007), applying 4000 iterations. 


\section{Results}

\subsection{Analysis of Behavioural Data}

\section{Fluency Performance and Spatial Distribution of Designs in the Five-Point Test}

Neglect patients and healthy controls showed significant differences in their Five-Point Test performance. Figural fluency (i.e., the number of produced designs) was significantly lower in neglect patients than in healthy controls (number of produced designs: healthy controls $\mathrm{m}=33.45, S D=$ 6.42; neglect patients $\mathrm{m}=12.26, S D=6.64 ; t(64)=12.03, p<.001)$.

Furthermore, neglect patients showed a strong rightward bias in their production of designs (as reflected by high, positive CoC values), whereas healthy controls showed a minimal leftward bias (as reflected by low, negative $\mathrm{CoC}$ values) (CoC values: healthy controls $\mathrm{m}=-0.04, S D=0.07$; neglect patients $\mathrm{m}=0.65, S D=0.29 ; t(56.11)=-15.07, p<.001)$.

More specifically, the analysis of the number of designs over the five columns of the test grid in the two groups revealed a significant main effect of the factor 'group' $(F(1,64)=144.82, p<.001)$, a significant main effect of the factor 'column' $(F(2.59,165.762)=24.79, p<.001)$, and, crucially, an highly significant interaction effect between the factors 'column $\mathrm{x}$ group' $(F(2.59,165.762)=$ 45.16, $p<.001)$. The mean number of produced designs for each column of the Five-Point Test grid in both groups is presented in Figure 5, showing a rightward gradient in neglect patients and a very modest leftward gradient in healthy controls.

Figure 5

about here 


\section{The Five-Point Test as a Diagnostic Tool for the Evaluation of Neglect}

A Pearson's correlation between the $\mathrm{CoC}$ of the cancellation tasks and the $\mathrm{CoC}$ of the Five-Point Test in neglect patients evidenced a highly significant $(r=.51, p<.001, n=46)$ and strong (i.e., $>$ .50 , according to (Cohen, 1988)) relationship, showing that the Five-Point Test is a valuable tool to measure neglect. Correlation between the CoC of the Five-Point Test and the Line Bisection task did not reveal any significance $(r=.197, p=.189, n=46)$.

In neglect patients, the $\mathrm{CoC}$ of the Five-Point Test showed a significantly stronger rightward deviation than the CoC of the cancellation tasks (CoC Five-Point Test $\mathrm{m}=0.65, S D=0.29$; CoC cancellation tasks $\mathrm{m}=0.32, S D=0.29 ; t(45)=-7.69, p<.001, n=46)$, suggesting that the Five-Point Test induced a larger spatial bias in neglect than the cancellation task.

A Pearson's correlation between the $\mathrm{CoC}$ in the Five-Point Test and the number of designs was highly significant $(r=-.573, p>0.001)$. Patients who produced more designs had a smaller CoC compared to patients with fewer designs.

\section{Perseverative Behaviour in the Five-Point Test}

Overall, neglect patients showed significantly more perseverative errors than healthy controls (percentage of perseverative errors: healthy controls $\mathrm{m}=8.59, S D=11.29$; neglect patients $=18.81, S D$ $=17.11 ; t(53.412)=-2.87, p=.006, n=66)$.

In neglect patients, the number of correct designs did not correlate with the number of perseverative errors $(r=.153, p=.220$, mean number of correct designs $=10.89(S D=6.7)$, mean number of perseverations $=2.41(S D=2.4))$. This means that participants who produced a higher number of correct designs in the Five-Point Test did not necessarily produce more perseverative errors.

The severity of neglect (reflected by the CoC) did not correlate with the number of perseverative errors (expressed as the percentage of all designs) $(r=0.13, p=0.40)$. This means that patients with 
a more positive $\mathrm{CoC}$ in the Five-Point Test (and hence a more severe neglect) did not necessarily show a higher percentage of perseverative errors.

Neglect patients produced significantly more Non-Neglect-driven perseverations than Neglectdriven perseverations (mean number of Neglect driven perseverations $\mathrm{m}=.50, S D=.84$; mean number of Non-Neglect-driven perseverations $=2.13, S D=2.25 ; t(45)=-5.262, p<0.001)$.

\section{Temporal Distribution of Perseverations}

In patients with perseverations $(n=32)$ the mean number of continuous perseverations $(m=1.75$, $S D=1.74)$ compared to the mean number of recurrent perseverations $(m=1.91, S D=1.49)$ was not significant $(t(31)=-.404, p=.689)$. On a descriptive level, we found 5 patients who produced the same designs continuously, 10 patients who produced recurrent perseverations, and 17 patients who produced both - continuous as well as recurrent perseverations. Patients produced between 0 and 5 different perseverative designs (Figure 6).

Figure 6

about here

\section{Spatial Distribution of Perseverations}

Overall, the mean spatial distribution of unique designs and perseverative designs did not differ in neglect patients with perseverative behaviour ( $\mathrm{CoC}$ unique designs: $\mathrm{m}=.60, S D=.34$; $\mathrm{CoC}$ perseverative designs: $\mathrm{m}=.67, S D=.31 ; t(31)=-1.40, p=.172, n=32)$. However, some patients showed a stronger rightward shift in the production of perseverations than in the production of unique designs. Thus, we divided patients presenting with perseverations into two subgroups: 1) patients with no rightward shift in perseveration production (i.e., $\mathrm{CoC}$ perseverations $\leq \mathrm{CoC}$ unique designs; $n=$ 
19); and 2) patients with a rightward shift in perseveration production (i.e., CoC perseverations > CoC unique designs; $n=13)$.

\subsection{Lesion-Symptom Mapping Analysis}

The VLSM analysis of continuous behavioural data (i.e., CoC of all design) using the BrunnerMunzel test yielded no significant results.Moreover, using a binomial Liebermeister test, we failed to find significant differences between the lesions of neglect patients with perseverations and neglect patients without perseverations. Descriptive subtraction plots (lesions of patients with perseverations minus patients without perseverations) are shown in Figure7.

Additional VLSM analysis using binomial Liebermeister test failed to find significant differences between neglect patients with continuous perseverations and patients with recurrent perseverations. In a further step, we contrasted the lesions of patients with a rightward shift in perseveration production (i.e., CoC perseverations > CoC unique designs) with those showing no rightward shift in perseveration production (i.e., $\mathrm{CoC}$ perseverations $\leq \mathrm{CoC}$ unique designs) by means of a Liebermeister test. This analysis revealed a significant lesion cluster (108 Voxels; corrected for false discovery rate [FDR; significance threshold of .05], 4000 permutations), located in the anterior part of the putamen (MNI coordinates 23, 11, 6; see Figure 8). This means that within the group of neglect patients presenting perseverative behaviour, those with a rightward shift in their production of perseverations are significantly more likely to have this putaminal area damaged than those who do not show this behaviour. 
Figure 7

about here

Figure 8

about here 


\section{Discussion}

In the present study, we demonstrate that the Five-Point Test, a well-known test to assess figural fluency, can also accurately measure perseverative behaviour and visual attention in neglect. Compared to healthy controls, neglect patients showed a significant spatial shift towards the right side in their design production, as well as significantly more perseverations. Whereas we did not find a significant correlation between number of perseverations and neglect severity per se, a VLSM analysis showed that - within the subgroup of patients with neglect and perseverative behaviour - the horizontal spatial distribution of these perseverations seems to critically depend on the integrity of the right putamen.

The Five-Point Test was found to be a valuable instrument to assess neglect. In comparison to healthy subjects, neglect patients produced significantly fewer designs, and their spatial distribution was significantly shifted towards the right side. Whereas healthy controls produced almost the same number of designs in each column, neglect patients showed a spatial gradient in their graphic production, i.e., the more towards the right the column of the test grid was located, the higher was the number of designs produced in this column. We also found a significant and strong correlation between the $\mathrm{CoC}$ of the Five-Point Test and the CoC of commonly applied cancellation tasks, suggesting clinical usefulness of the Five-Point Test in neglect assessment. Interestingly, the CoC of the Five-Point Test showed a significantly stronger rightward deviation than the CoC of the cancellation tasks. This result suggests that the Five-Point Test might induce a larger spatial bias than commonly used cancellation tasks.

Moreover, the Five-Point Test allows to easily and reliably ascertain the presence, the severity, and the spatial dynamics of perseverative behaviour in neglect patients (in terms of number and spatial distribution of perseverative graphic productions). In previous studies using cancellation tasks, no univocal definition of perseverative errors has been used (Gandola, et al., 2013). By contrast, perseverations are clearly defined in the Five-Point Test. 
Regarding fluency, we found that neglect patients produced significantly fewer designs than healthy subjects. Furthermore, patients with a more severe neglect produced less designs. This impaired fluency might be explained by a reduction of arousal, which is an important component of severe neglect following right hemisphere injury (Corbetta \& Shulman, 2011; Heilman, Valenstein, \& Watson, 2000).

Some previous studies have suggested no relationship between neglect severity and perseverative behaviour (Pia, et al., 2009; Pia, et al., 2013; Rusconi, et al., 2002; Vallar, et al., 2006). In line with these results, in our study we did not find any correlation between neglect severity, as measured with the Five-Point Test, and the number of perseverations produced in the same test. Overall, patients produced significantly more perseverations that occurred on the left-hand of the original design than perseverations that occurred on the right-hand of the original designs.

Lesions in patients with neglect and additional perseveration also tended to be located around the well-known inhibition network, involving the inferior frontal gyrus (IFG), the dorsolateral prefrontal cortex (DLPFC) and the premotor cortex (Gandola, et al., 2013; Husain \& Kennard, 1997; Mannan, et al., 2005; Menon, et al., 2001; Pierrot-Deseilligny, et al., 1991). This suggest that impaired inhibition, or impaired 'contention scheduling' with a failure for controlling action selection (Cooper \& Shallice, 2000) may play a pivotal role.

However, additional analysis revealed that in some neglect patients, the production of perseverations showed a stronger rightward shift than the production of unique designs. This suggests that, at least in a subsample of patients, perseverations are influenced by a visual attentional gradient, as suggested by some authors (Kleinman, et al., 2013; Nys, et al., 2006). Indeed, a lesion analysis with a VLSM approach showed that the spatial distribution of perseverative responses in neglect critically depends on the integrity of the right putamen. Neglect patients with a putaminal lesion showed a stronger rightward shift in perseverations production compared to those without a lesion in this region. 
The putamen has previously been described as a hub, connecting the networks subtending the control of inhibition and visual attention (Jarbo \& Verstynen, 2015). On the one hand, the putamen is thought to be a central component of the frontal-subcortical circuit associated with inhibitory processes of executive control (van Belle, et al., 2014). For instance, a recent study using fibre tractography (Jarbo \& Verstynen, 2015) has evidenced both structural and functional connectivity between the putamen and the inferior frontal gyrus, a cortical area strongly implied in inhibitory functions (Jahanshahi, et al., 2015). In fact, a lesion of the putamen, triggering a disruption of the frontalsubcortical circuit, leads to impaired executive functioning with perseverations (Kokubo, Suzuki, Hattori, Miyai, \& Mori, 2015).

On the other hand, the putamen also has anatomical connections with rostral parietal areas, thereby influencing visual attention (Jarbo \& Verstynen, 2015). Thus, a lesion of the putamen can not only lead to perseverations, but also to impaired contralesional visual attention. Indeed, previous studies demonstrated that patients with a stroke affecting the putamen show left-sided neglect (Karnath \& Rorden, 2012; Vallar \& Perani, 1987).

Alternatively, perseverative behaviour in neglect has been discussed in the context of impaired working-memory processes (Husain, Mannan, Hodgson, Wojciulik, Driver \& Kennard, 2001). Especially, recurrent perseverations have been mentioned in the context of memory impairments (Helm-Estabrooks, N., Ramage, A., Bayles, K. A. \& Cruz, R.,1998; Sandson \& Albert , 1987). In our study patients did not show any difference between the mean number of continuous, i.e. repetitive designs directly adjoining to the same designs, and the mean number of recurrent, i.e. repetitive designs not adjoining to the same designs, perseverations. Furthermore, VLSM analyses between patients with continuous perseverations and patients with recurrent perseverations were not significant. However, neglect patients produced more perseverations on the left-hand side of the original design than the right-hand (i.e. patients had significantly more non-neglect driven than neglectdriven perseverations), which might be an additional argument for impaired working memory pro- 
cesses. Hence, beside impaired response inhibition an impaired working-memory may influence perseverative behaviour.

In summary, our findings suggest that the relationship between impairments in visual attention and response inhibition might not be a direct one (for instance, neglect severity did not correlate with perseverative behaviour). Rather, it seems that the influence of visual attention impairment on perseverations may be conveyed through a 'moderator entity', for which the putamen appears to be a good candidate as a neural substrate. It is therefore conceivable that the rightward shift in perseveration production, observed in the present study in neglect patients with a lesion involving the putamen, represents an additional spatial gradient, i.e., over and above the one determining neglect. Such a hypothesis is in line with the view that the putamen not only plays a key role in the modulation of motor functions, but also of cognitive functions (Haber, 2003) see also for a review Provost, Hanganu, \& Monchi, 2015).

Our study also has some limitations. First, we did not include patients with right-hemispheric lesions but no neglect. Thus, we cannot compare the results of our sample with the ones of a control group with perseverations but no neglect. As such, our results cannot be generalized to perseverative behaviour per se, but are - in line with the main focus of our study - specific to the occurrence of perseverative behaviour in neglect patients. Future studies, including a larger sample of patients with right hemispheric lesions, with and without neglect, should shed further light on more general mechanisms involved in perseveration. )

Second, the administration of the Five-Point Test and of the cancellation task shows some methodological differences. The Five-Point test requires participants to 'actively' produce designs, while the Cancellation Tasks asks for cancelling targets that are already present. Furthermore, patients are given three minutes of time to complete the Five-Point Test, whereas cancellation tasks are generally untimed. Therefore, a direct comparison of the sensitivity of the two tests might be confounded through this factor. However, one may argue that, even when administering the cancellation tasks 
on A3 sheets of paper, in landscape orientation (which, because of the greater horizontal extension, may result in a greater difficulty), we found the Five-Point Test (administered on A4 sheets of paper, in portrait orientation) to be more sensitive in assessing neglect. Furthermore, an allocentric perceptual impairment may also influence the performance in the Five-Point Test. Allocentric neglect cannot be reliably assessed with the cancellation tasks used in the present study (i.e., The Bells test (Gauthier, Dehaut, \& Joanette, 1989), the Star Cancellation test (Wilson, et al., 1987), or the Random Shape Cancellation test (Weintraub \& Mesulam, 1988)).

In conclusion, we found that the Five-Point Test is able to reliably assess neglect as well as perseverative behaviour. Furthermore, our study contributes to the understanding of the interplay between executive processes, which should prevent perseverative phenomena, and neglect. Our lesion analysis approach showed that the spatial distribution of perseverations significantly depends on the integrity of the right putamen, suggesting that this region might play a role for the complex integration between visual attention and response inhibition processes. 


\section{Acknowledgements}

This work was supported by SNF Grant Nr 320030_169789.

We are grateful to the patients and healthy individuals who took part in our study. We would also

like to thank the clinical teams at the Inselspital, Bern University Hospital and at the Kantonsspital Luzern for their assistance.

The authors declare that they have no conflict of interest. 


\section{References}

Bari, A., \& Robbins, T. W. (2013). Inhibition and impulsivity: behavioral and neural basis of response control. Prog Neurobiol, 108, 44-79.

Cohen, J. (1988). Statistical power analysis for the behavioral sciences (2nd ed.) (2 ed.).

Corbetta, M., \& Shulman, G. (2002). Control of goal-directed and stimulus-driven attention in the brain. Nat Rev Neurosci., 3, 201-205.

Corbetta, M. \& Shulman, G. L. (2011). Spatial Neglect and Attention Networks. Annu Rev Neurosci, 34, 569-599.

Gandola, M., Toraldo, A., Invernizzi, P., Corrado, L., Sberna, M., Santilli, I., Bottini, G., \& Paulesu, E. (2013). How many forms of perseveration? Evidence from cancellation tasks in right hemisphere patients. Neuropsychologia, 51, 2960-2975.

Gauthier, L., Dehaut, F., \& Joanette, Y. (1989). The Bells test: A quantitative and qualitative test for visual neglect. International Journal of Clinical Neuropsychology, 11, 49-54.

Head, D., Kennedy, K. M., Rodrigue, K. M. \& Raz, N. (2009). Age-Differences in Perseveration: Cognitive and Neuroanatomical Mediators of Performance on the Wisconsin Card Sorting Test. Neuropsychologia, 47 (4), 1200-1203.

Heilman, K. M., Valenstein, E. \& Watson, R. T. (2000). Neglect and Related Disorders. Seminars in Neurology, 20 (4), 463- 470.

Helm-Estabrooks, N., Ramage, A., Bayles, K. A. \& Cruz, R. (1998). "Perseverative Behaviour in Fluent and Non-Fluent Aphasic Adults." Aphasiology, 12, (7), 689-698.

Husain, M., \& Kennard, C. (1997). Distractor-dependent frontal neglect. Neuropsychologia, 35, 829-841.

Husain, M., Mannan, S., Hodgson, T., Wojciulik, E., Driver, J., \& Kennard, C. (2001). Impaired spatial working memory across saccades contributes to abnormal search in parietal neglect. Brain, 124, 941-952.

Jahanshahi, M., Obeso, I., Rothwell, J. C., \& Obeso, J. A. (2015). A fronto-striato-subthalamicpallidal network for goal-directed and habitual inhibition. Nature Reviews Neuroscience, 16, 719-732.

Jarbo, K., \& Verstynen, T. D. (2015). Converging Structural and Functional Connectivity of Orbitofrontal, Dorsolateral Prefrontal, and Posterior Parietal Cortex in the Human Striatum. Journal of Neuroscience, 35, 3865-3878.

Karnath, H. O., Rennig, J., Johannsen, L., \& Rorden, C. (2011). The anatomy underlying acute versus chronic spatial neglect: a longitudinal study. Brain, 134, 903-912.

Karnath, H. O., \& Rorden, C. (2012). The anatomy of spatial neglect. Neuropsychologia, 50, 10101017. 
Kimberg, D. Y., Coslett, H. B., \& Schwartz, M. F. (2007). Powerinvoxel- based lesionsymptommapping. J. Cogn.Neurosci., 19, 1067-1080.

Kleinman, J. T., DuBois, J. C., Newhart, M., \& Hillis, A. E. (2013). Disentangling the neuroanatomical correlates of perseveration from unilateral spatial neglect. Behav Neurol., 26, 131138.

Kokubo, K., Suzuki, K., Hattori, N., Miyai, I., \& Mori, E. (2015). Executive Dysfunction in Patients with Putaminal Hemorrhage Journal of Stroke and Cerebrovascular Diseases, 24, 1978-1985.

Mannan, S. K., Mort, D. J., Hodgson, T. L., Driver, J., Kennard, C., \& Husain, M. (2005). Revisiting previously searched locations in visual neglect: role of right parietal and frontal lesions in misjudging old locations as new. J Cogn Neurosci, 17, 340-354.

Mark, V. W., Kooistra, C. A., \& Heilman, K. M. (1988). Hemispatial neglect affected by nonneglected stimuli. Neurology, 38, 1207-1211.

Medina, J., Kimberg, D. Y., Chatterjee, A., \& Branch Coslett, H. (2010). Inappropriate usage of the Brunner-Munzel test in recent voxel-based lesion-symptom mapping studies. Neuropsychologia, 48, 341-343.

Menon, V., Adleman, N. E., White, C. D., Glover, G. H., \& Reiss, A. L. (2001). Error-related brain activation during a Go/NoGo response inhibition task. Hum Brain Mapp, 12, 131-143.

Na, D. L., Adair, J. C., Kang, Y., Chung, C. S., Lee, K. H., \& Heilman, K. M. (1999). Motor perseverative behavior on a line cancellation task. Neurology, 52, 1569 - 1576.

Nachev, P., Coulthard, E., Jäger, H. R., Kennard, C., \& Husain, M. (2008). Enantiomorphic normalization of focally lesioned brains. NeuroImage, 39, 1245-1226.

Nys, G. M., van Zandvoort, M. J., van der Worp, H. B., Kappelle, L. J., \& de Haan, E. H. (2006). Neuropsychological and neuroanatomical correlates of perseverative responses in subacute stroke. Brain, 129, 2148-2157.

Pia, L., Folegatti, A., Guagliardo, M., Genero, R., \& Gindri, P. (2009). Are drawing perseverations part of the neglect syndrome? Cortex, 45, 293-292.

Pia, L., Ricci, R., Gindri, P., \& Vallar, G. (2013). Drawing perseveration in neglect: effects of target density. J Neuropsychol, 7, 45-57.

Pierrot-Deseilligny, C., Rivaud, S., Gaymard, B., \& Agid, Y. (1991). Cortical control of reflexive visually-guided saccades. Brain, 114, 1473-1485.

Provost, J.-S., Hanganu, A., \& Monchi, O. (2015). Neuroimaging studies of the striatum in cognition Part I: healthy individuals. Frontiers in Systems Neuroscience, 9.

Regard, M., Strauss, E., \& Knapp, P. (1982). Children's production on verbal and non-verbal fluency tasks. . Perceptualand Motor Skills, 55, 839-844.

Ronchi, R., Posteraro, L., Fortis, P., Bricolo, E., \& Vallar, G. (2009). Perseveration in left spatial neglect: Drawing and cancellation tasks. Cortex, 45, 300-312. 
Rorden, C., \& Karnath, H. O. (2010). A simple measure of neglect severity. Neuropsychologia, 48, 2758-2763.

Rorden, C., Karnath, H. O., \& Bonilha, L. (2007). Improving Lesion-Symptom Mapping. J Cogn Neurosci, 19, 1081-1088.

Rusconi, M. L., Maravita, A., Bottini, G., \& Vallar, G. (2002). Is the intact side really intact? Perseverative responses in patients with unilateral neglect: a productive manifestation. $\mathrm{Neu}$ ropsychologia, 40.

Sandson, J., \& Albert, M. L. (1987). Perseveration in behavioral neurology. Neurology, 37, 17361174.

Tucha, L., Aschenbrenner, S., Koerts, J. \& Lange, K. W. (2012). The Five-Point Test : Reliability, Validity and Normative Data for Children and Adults, PLOSONE.

Vallar, G., \& Perani, D. (1987). The anatomy of spatial neglect in humans. Advances in Psychology, $45,235-258$.

Vallar, G., Zilli, T., Gandola, M., \& Bottini, G. (2006). Productive and defective impairments in the neglect syndrome: graphic perseveration,drawing productions and optic prism exposure. Cortex, 42, 911-920.

van Belle, J., Vink, M., Durston, S., \& B. Zandbelt, B. B. (2014). Common and unique neural networks for proactive and reactive response inhibition revealed by independent component analysis of functional MRI data. Neuroimage, 103, 65-74.

Vossel, S., J. Geng, J. J., \& Fink, G. R. (2014). Dorsal and Ventral Attention Systems: Distinct Neural Circuits but Collaborative Roles. The Neuroscientist, 20(2), 150-159.

Weintraub, S., \& Mesulam, M. (1988). Visual hemispatial inattention: stimulus parameters and exploratory strategies. Journal of Neurology, Neurosurgery, and Psychiatry, 51, 1481-1488.

Wilson, B. A., Cockburn, J., \& Halligan, P. W. (1987). Development of a behavioral test of visuospatial neglect. Archives of Physical Medicine and Rehabilitation, 68, 98-102. 
File Name "Figure 1 Brain lesions of all 46 patients" 2 column fitting image

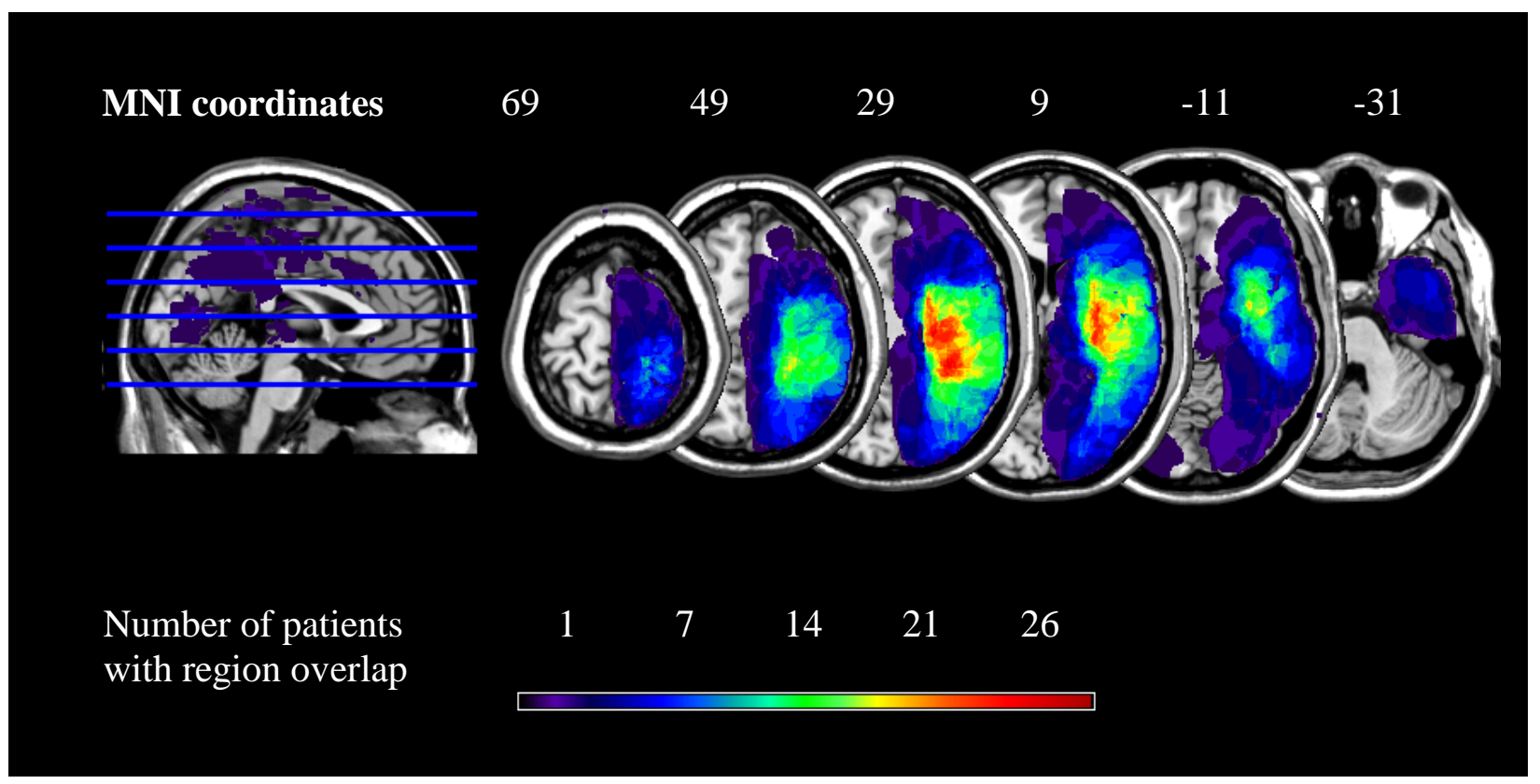

Figure 1 Brain lesions of all 46 patients with right-hemisphere stroke. The color-coded legend is determined by the number of patients with damage to a specific brain region. Lesion overlap maps are plotted on the $\mathrm{CH} 2$ template available in MRIcron (http://sph.sc.edu/comd/rorden/mricron). Axial slices are oriented according to the neurological convention. The z-position of each axial slice, in MNI coordinates, is indicated by the numbers at the top of the figure, and also depicted by the blue lines on the sagittal slice (left-hand side of the figure). 
File Name "Figure 2 Neglect driven and Non-Neglect driven perseverations" single column fitting image

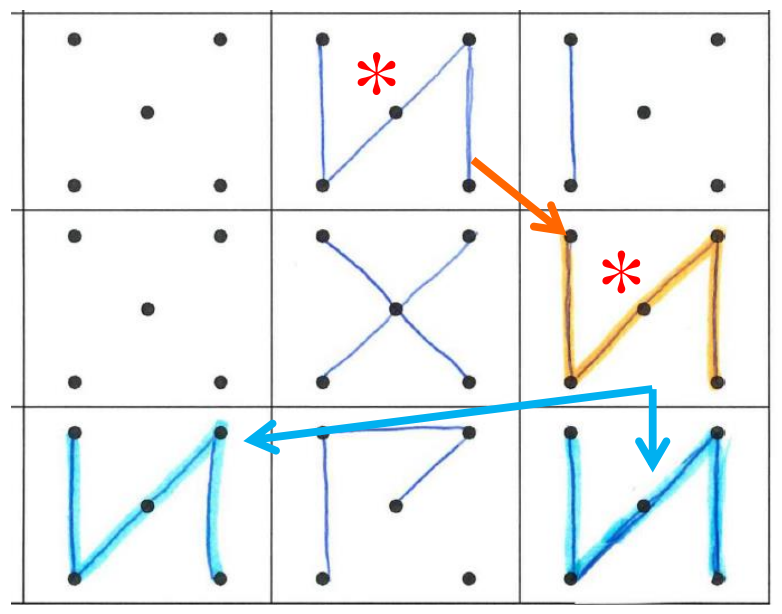

Figure 2 Neglect-driven perseverations (orange) were repetitive designs, which occur in a column that is on the right-hand of an original design with the same pattern. Non-Neglect-driven perseverations (blue) were defined as perseverations which occur in a column that is on the left-hand or below of a previous design (highlighted with a red star) with the same pattern. 
$\underline{\text { File Name "Figure } 3 \text { Continuous and Recurrent Perseverations" single column fitting image }}$

\section{Continuous}

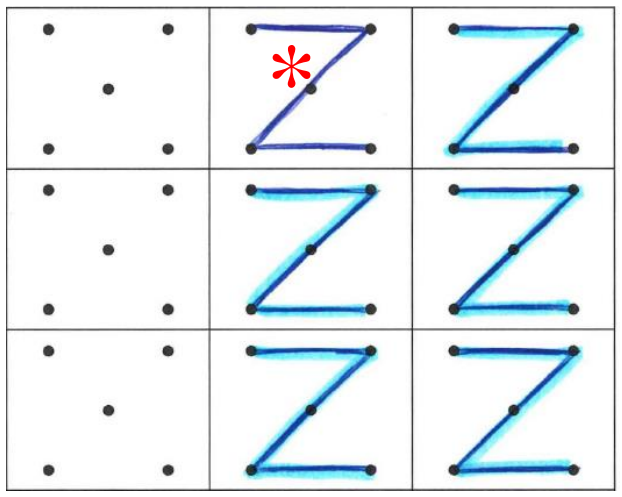

\section{Recurrent}

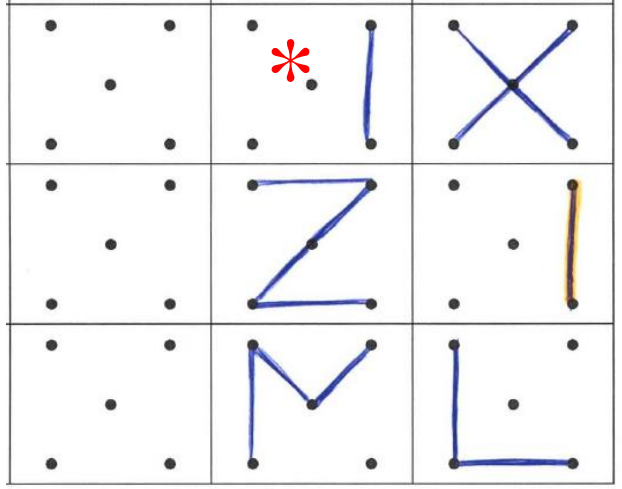

Figure 3 Continuous perseverations (blue) were defined as repetitive designs in directly adjoining rectangles (here as an example $\mathrm{Z}$ ). Recurrent perseverations (orange) were defined as repetitive designs in not adjoining rectangles (here as an example I). The original designs are highlighted with a red star. 
File Name "Figure 4 Example of the results of the 5PT" single column fitting image

(A)

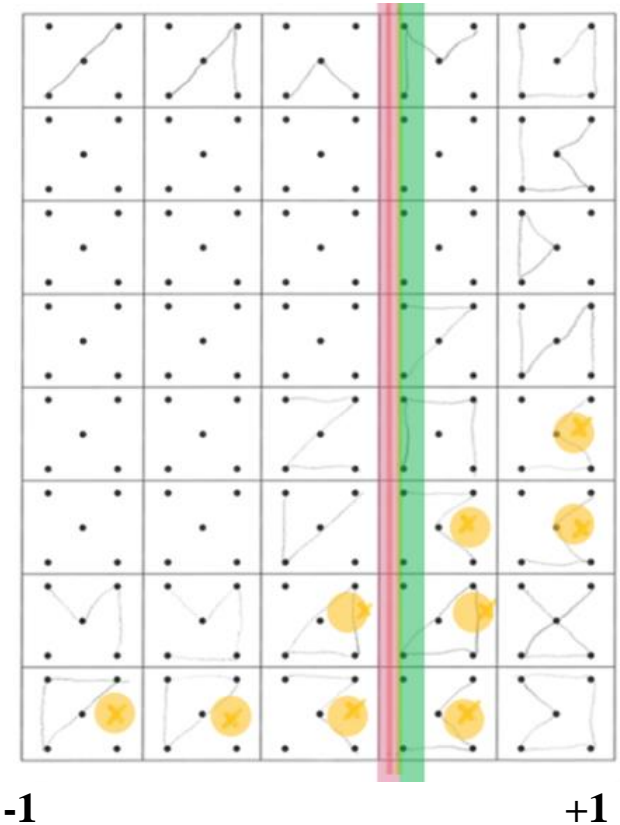

(B)

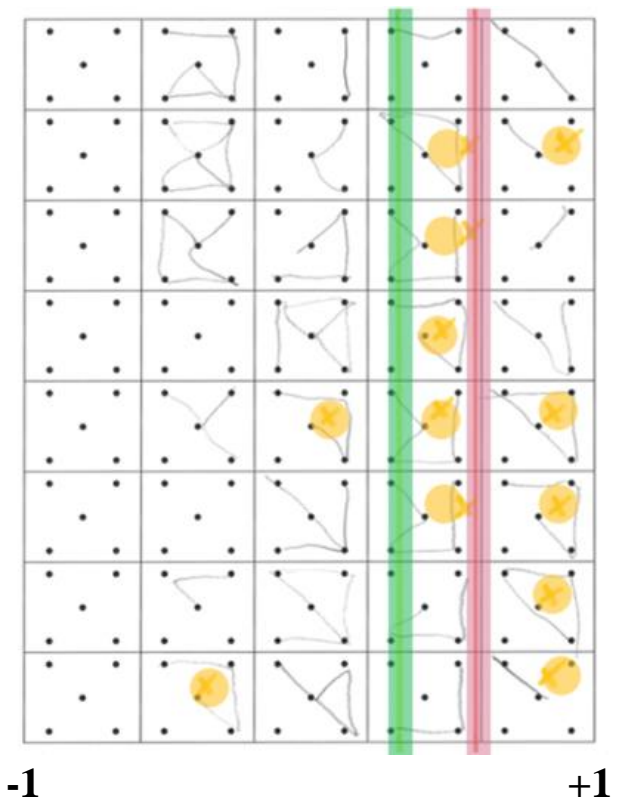

Figure 4 Example of the results of the Five-Point Test in: (A) a patient with left hemispatial neglect, but no rightward shift in perseverations production (Centre of Cancellation (CoC) unique designs $=0.258 ; \mathrm{CoC}$ of perseverations $=0.225) ;(\mathrm{B})$ a patient with left hemispatial neglect and a rightward shift in perseverations production $(\mathrm{CoC}$ unique designs $=0.296$; $\mathrm{CoC}$ of perseverations $=$ 0.575). Unfabled designs represent unique designs. Perseverative errors (i.e., repeated designs) are highlighted by yellow dots. The green lines represent the $\mathrm{CoC}$ of unique designs, the red lines the $\mathrm{CoC}$ of perseverations. The $\mathrm{CoC}$ can range from -1 to +1 (see numbers at the bottom of the grids); positive values indicate a shift towards the right side of space, negative values indicate a shift towards the left side of space. A CoC value of 0 indicates an unbiased spatial distribution. 
File Name "Figure 5 ANOVA 5PT" single column fitting image

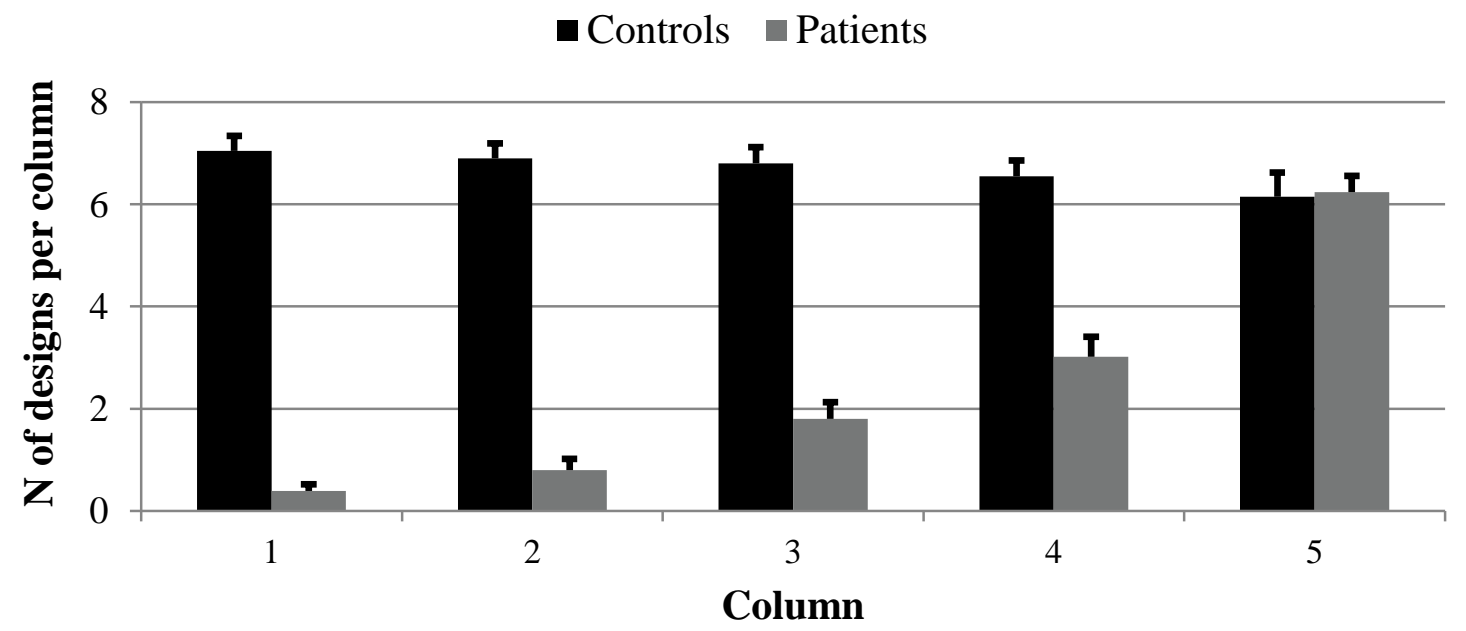

Figure 5. Mean number of produced designs for each column of the Five-Point Test grid, in neglect patients (grey bars) and in healthy controls (black bars). Columns are numbered from the left to the right with ascending numbers (i.e., $1=$ far left; $3=$ middle; $5=$ far right). Error bars indicate the standard error of means. 
File Name "Figure $6 \mathrm{Nr}$ of participants and nr of different perseverative designs" single column fitting image

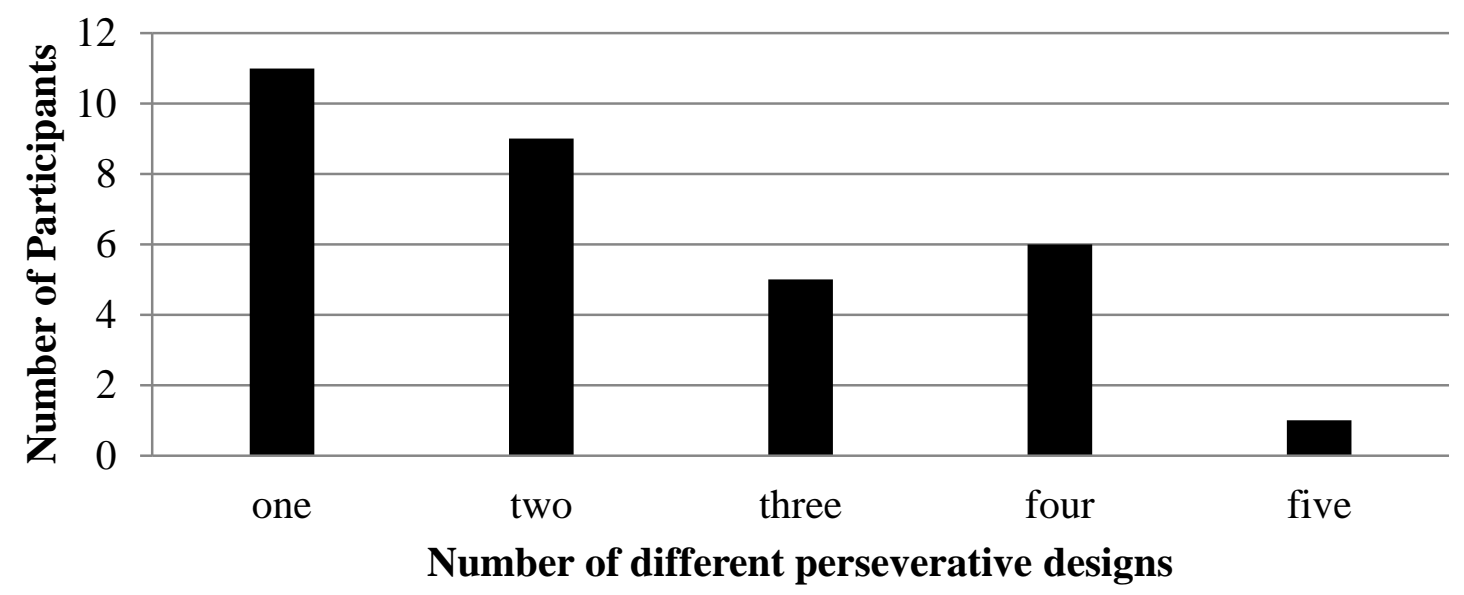

Figure 6 Number of participants who produced one, two, three, four or five different perseverative designs in the Five-Point Test. 
File Name "Figure 7 Brain Lesions Pat with and without perseverations" 2 column fitting image

A) Brain lesions of patients with perseverations $(n=32)$

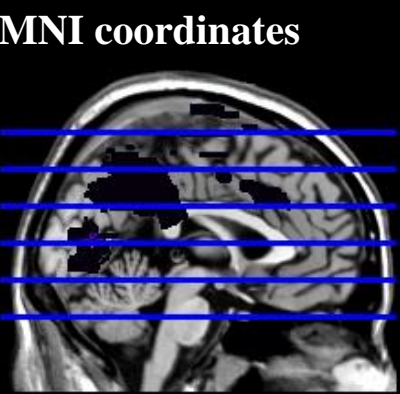

29

9

$-11 \quad-31$

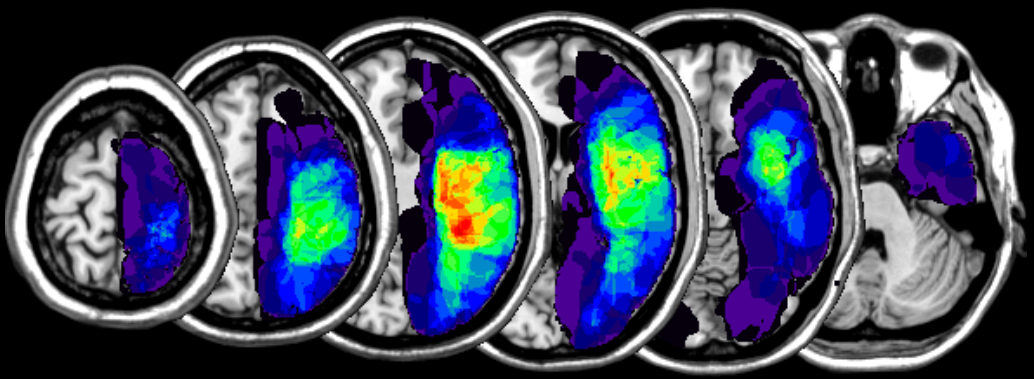

Number of patients with region overlap

5

10

15

20

B) Brain lesions of patients without perseverations $(n=14)$
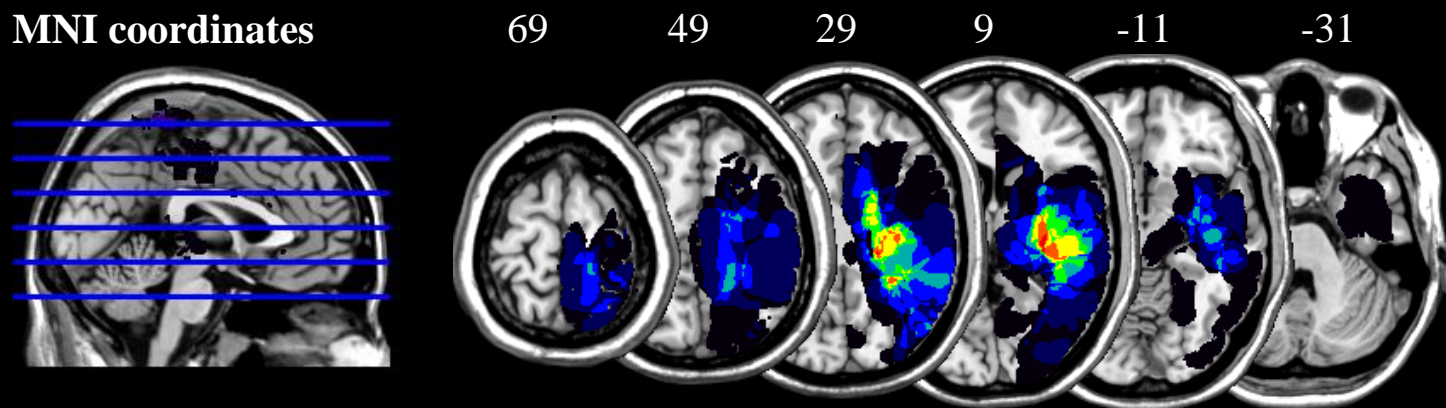

Number of patients

2

with region overlap

C) Lesion subtraction plots between patients with perseverations $(n=32)$ and patients without perseverations $(n=14)$
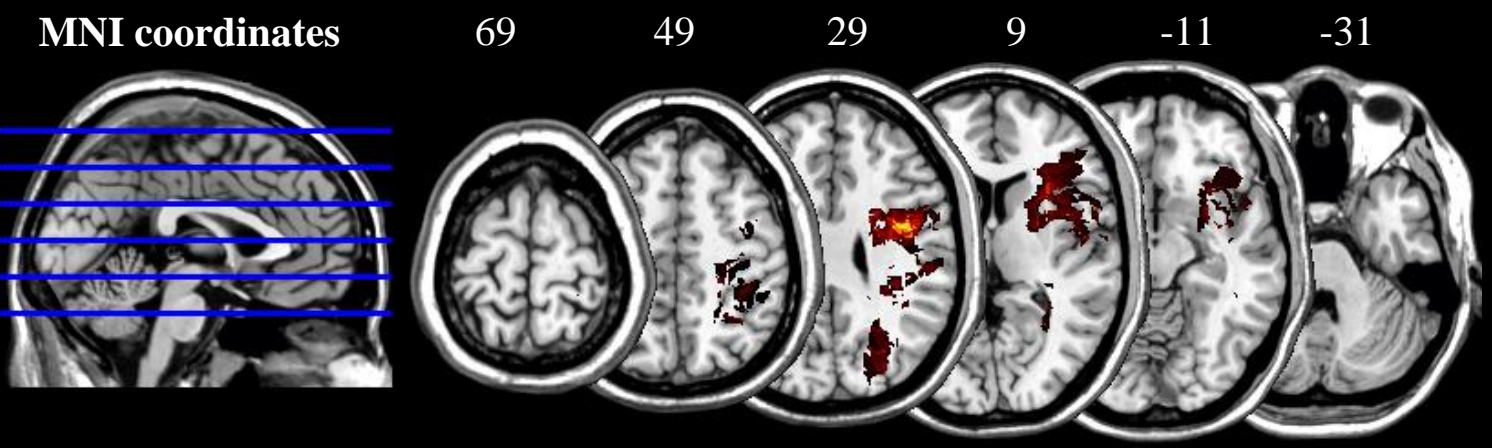

49

29

8

difference percent

24

30

36

42

overlap 
Figure 7. (A) Brain lesions of all 32 Patients with perseverations; (B) Brain lesions of all 14 patients without perseverations; (C) Lesion subtraction plots between patients with perseverations $(n$ $=32$ ) and patients without perseverations $(n=14)$. The color-coded legend is determined by the number of patients with damage to a specific brain region. Lesion overlap maps are plotted on the $\mathrm{CH} 2$ template available in MRIcron (http://sph.sc.edu/comd/rorden/mricron). Axial slices are oriented according to the neurological convention. The z-position of each axial slice, in MNI coordinates, is indicated by the numbers at the top of the figure, and also depicted by the blue lines on the sagittal slice (left-hand side of the figure). 
File Name "Figure 8 Putamen" 2 column fitting image

(A) Brain lesions of patients with a rightward shift in perseveration production $(n=13)$
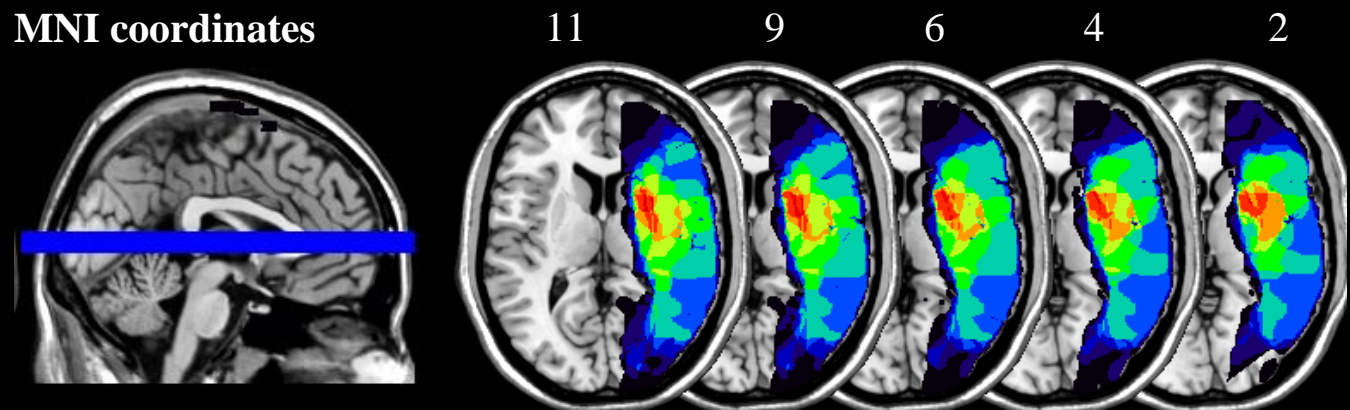

Number of patients with region overlap

(B) Brain lesions of patients with no rightward shift in perseveration production $(n=19)$
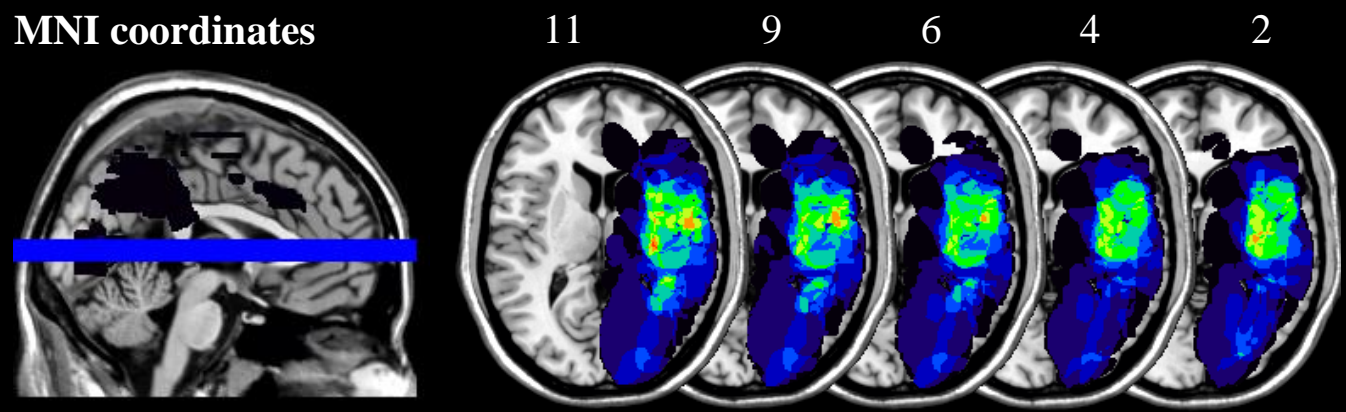

Number of patients with region overlap

$$
\begin{array}{lllll}
2 & 4 & 6 & 8 & 10
\end{array}
$$

(20)

(C) Lesion contrast of patients with a rightward shift in perseveration production with those showing no rightward shift in perseveration production

MNI coordinates

6

2
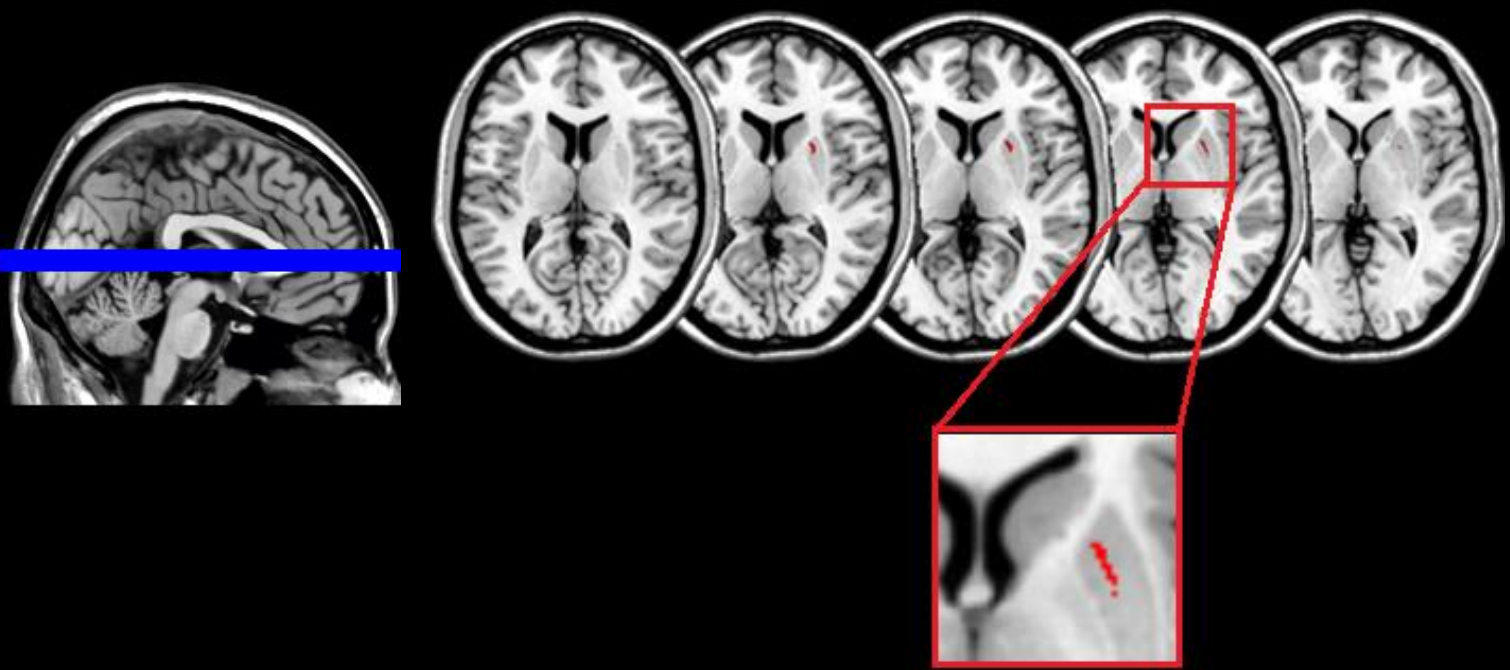
Figure 8. A) Brain lesions of all 13 patients with a rightward shift in perseveration production (i.e., CoC perseverations > CoC unique designs); (B) Brain lesions of all 19 patients with no rightward shift in perseveration production (i.e., CoC perseverations $\leq \mathrm{CoC}$ unique designs); (C) Results of the VLSM analysis concerning the rightward shift in perseveration production in neglect patients. Voxels with damage that were a significant predictor of a rightward shift in perseveration production are depicted in red (significance level 0.05, based on the Liebermeister test with FDR correction). The significant lesion cluster of 108 voxels was located in the anterior putamen (MNI coordinates: 23, 11, 6). The cluster of voxels is displayed on the $\mathrm{CH} 2$ template in MNI space, as available in MRIcron (http://sph.sc.edu/comd/rorden/mricron). Axial slices are oriented according to the neurological convention. The z-position of each axial slice, in MNI coordinate is indicated by the numbers at the top of the figure, and also depicted by the blue lines on the midsagittal slice (left-hand side of the figure). 